\title{
Produção de leite, leite residual e concentração hormonal de vacas Gir $\times$ Holandesa e Holandesa em ordenha mecanizada exclusiva
}

\author{
[Milk yield, residual milk, oxytocin concentration and cortisol release in Holstein and \\ Gir $\times$ Holstein cows machine milked without stimulus from a calf] \\ M.A.F. Porcionato ${ }^{1}$, J.A. Negrão ${ }^{2}$, M.L.P. Lima ${ }^{3}$ \\ ${ }^{1}$ Doutorando em Zootecnia - Faculdade de Ciências Agrárias e Veterinária - UNESP \\ Via de Acesso Prof. Paulo Donato Castellane, s/n \\ 14884-900 - Jaboticabal, SP \\ ${ }^{2}$ Faculdade de Zootecnia e Engenharia de Alimentos - USP - Pirassununga, SP \\ ${ }^{3}$ Instituto de Zootecnia - Ribeirão Preto, SP
}

\begin{abstract}
RESUMO
Avaliaram-se as concentrações de ocitocina e de cortisol e as produções de leite e de leite residual de três grupos genéticos de vacas: $1 / 2$ Gir $\times 1 / 2$ Holandês $(n=6$, grupo genético $F 2$ ), $1 / 4 \mathrm{Gir} \times 3 / 4$ Holandês $(n=6$, grupo genético F3) e Holandês ( $\mathrm{n}=6$, grupo genético $\mathrm{H}$ ), ordenhadas mecanicamente e na ausência do bezerro. $\mathrm{O}$ grupo genético $\mathrm{H}$ produziu mais leite do que os grupos $\mathrm{F} 2$ e F3, e a concentração de ocitocina foi semelhante nos três grupos. $\mathrm{O}$ aumento de ocitocina durante a ordenha foi maior para os grupos $\mathrm{H}$ e F3 em relação ao grupo F2. O leite residual do grupo F2 foi maior do que o dos grupos genéticos F3 e H. O cortisol do grupo F2 foi maior do que o das vacas dos demais grupos genéticos. Vacas dos grupos F2 e F3 foram mais estressadas durante a ordenha do que vacas do grupo $\mathrm{H}$, mas as dos dois grupos genéticos cruzados liberavam suficiente quantidade de ocitocina que permitiu a ejeção efetiva de leite.
\end{abstract}

Palavras-chave: bovino, ordenha mecânica, ejeção de leite, Gir $\times$ Holandesa

\begin{abstract}
In a machine milking system in which calves were not present, oxytocin concentration (OT), cortisol release (CORT), milk yield and residual milk were evaluated for cows of three genetic groups: $1 / 2$ Gir $\times 1 / 2$ Holstein $(n=6$, genetic group F2), 1/4Gir $\times 3 / 4$ Holstein $(n=6$, genetic group F3) and Holstein $(n=6$, genetic group $H$ ). Group $H$ had higher milk yield than groups $F 2$ and $F 3$, whereas OT was similar among groups. The increase in OT during milking was greater for $\mathrm{H}$ and $\mathrm{F} 3$ than for F2. Residual milk for F2 was higher than for F3 and H. The CORT for F2 was higher than for cows of the other two genetic groups. Cows from F2 and F3 were more stressed than H cows during machine milking, but the Gir $\times$ Holstein groups did release sufficient OT to induce an effective milk letdown response.
\end{abstract}

Keywords: cattle, behavior, milk ejection, stress

Recebido para publicação em 14 de abril de 2004

Recebido para publicação, após modificações, em 9 de novembro de 2004

E-mail: 1elo10@hotmail.com 


\section{INTRODUÇÃO}

Vacas da raça Gir e touros da raça Holandesa têm sido cruzados para melhorar a produção de leite nos trópicos (Madalena e Trivedi, 1998; Sandoval-Castro et al., 1999), concomitante à criação de vacas Gir continuamente selecionadas para a produção de leite (Magnabosco et al., 1993; Souza et al., 1996). Contudo, vacas da raça Gir e seus cruzamentos, muitas vezes, são pouco adaptadas à ordenha mecanizada.

Alguns produtores mantêm vacas mestiças (Gir x Holandesa) amamentando seus bezerros, pois, assim, produzem mais leite com o bezerro ao pé do que quando ordenhadas sem o bezerro (Negrão e Marnet, 2003). Outros produtores desmamam os bezerros ao nascimento Essa decisão negligencia o fato de que a desmama é acompanhada de uma importante diminuição da produção de leite (Tancin et al., 1995; Marnet e Negrão, 2000).

Embora algumas raças zebuínas apresentem baixa persistência de lactação (Abubakar e Buvanendran, 1981; Murugaiyah et al., 2001) e retenham volume alto de leite residual (Murugaiyah et al., 2001), recentemente foi demonstrado que vacas Gir x Holandesa liberaram ocitocina durante a ordenha semelhante à liberação durante a amamentação/ordenha combinadas (Negrão e Marnet, 2002), sugerindo que as vacas desse grupo genético podem ser submetidas à ordenha mecânica exclusiva.

O objetivo deste trabalho foi estudar em diferentes grupos raciais $(\mathrm{Gir} \times$ Holandesa e Holandesa) a ação da ordenha mecanizada exclusiva sobre a liberação de ocitocina e cortisol, sobre a produção de leite e leite residual, bem como sobre o comportamento durante a ordenha.

\section{MATERIAL E MÉTODOS}

O experimento foi conduzido no início do verão, utilizando-se seis vacas $1 / 2$ Gir $\times$ Holandesa (grupo F2), seis $1 / 4$ Gir x Holandesa (grupo F3) e seis da raça Holandesa (grupo $\mathrm{H}$ ), na mesma ordem de lactações. As vacas sob pastejo tiveram livre acesso à água (Panicum maximum) e ao suplemento mineral-vitamínico. Antes de cada ordenha, receberam mistura de grãos (milho, farelo de soja e sorgo) de acordo com a produção de leite (Nutrient..., 2001). Foram ordenhadas duas vezes ao dia (às 6 e às 18 horas).

A produção de leite foi medida a cada ordenha, durante todo o experimento, e o leite residual foi medido ao final da ordenha, após cinco minutos da administração de $5 \mathrm{ml}$ de ocitocina intramuscular (5UI).

Após o parto, as vacas foram separadas dos bezerros e ordenhadas mecanicamente durante toda a lactação. Foram colhidas amostras de sangue, via veia jugular, na ordenha da manhã dos dias 40, 41, 42, 43, 44 e 45 da lactação, 3 minutos antes e $1,2,3,5,7,10$ e 15 minutos após o início da ordenha. Colhidas em tubos heparinizados e imediatamente centrifugadas a $4^{\circ} \mathrm{C}$ a $1500 \mathrm{xg}$ por 15 minutos, as amostras de plasma foram estocadas a $-20^{\circ} \mathrm{C}$ até o momento do uso.

A concentração de ocitocina (OT) foi determinada pelo método imunoenzimático (Marnet et al., 1998), e a concentração plasmática de cortisol (CORT) determinada em kits de dosagem imunoenzimáticas ${ }^{1}$.

A avaliação de comportamento dos animais foi feita durante a ordenha da manhã, do $60^{\circ}$ ao $75^{\circ}$ dia de lactação, por meio de entrevistas com os ordenhadores, que responderam a um questionário após cada ordenha.

Empregou-se programa SAS (User's... 2000) para as análises das médias de produção de leite e leite residual. A probabilidade utilizada foi $\mathrm{P}<0,05$, e todos os valores apresentados estão representados pela média e o respectivo erro padrão. $\mathrm{O}$ modelo estatístico considerou o efeito dos grupos (3), a identidade do animal (18), os dias de ordenhas (6), os tempos de amostragem (8) e as interações.

Para descrever o efeito dos grupos na liberação total dos hormônios durante as ordenhas, analisou-se a área sob a curva corrigida para as concentrações base. A correlação entre a produção de leite, as concentrações hormonais durante a ordenha, bem como a liberação total dos hormônios, foram avaliadas pela correlação

${ }^{1}$ Diagnostic System Laboratories, Inc. 
de Pearson. As freqüências do comportamento foram analisadas pelo teste de qui-quadrado e apresentadas em representação gráfica.

\section{RESULTADOS}

Os perfis dos hormônios OT e CORT, para os diferentes grupos raciais, são mostrados nas Fig. 1 e 2, respectivamente. A concentração de OT antes da ordenha $(-3 \mathrm{~min})$ foi mais alta $(\mathrm{P}<0,05)$ nos grupos F2 e F3. Para todos os grupos, a concentração de OT antes da ordenha foi mais baixa do que a concentração após o início da ordenha $(\mathrm{P}<0,05)$. Os perfis dos hormônios mostraram-se diferentes, isto é, o aumento da OT foi mais rápido durante a ordenha nos grupos $\mathrm{He}$ F3 (1 e 2 minutos, respectivamente) do que no grupo F2 (3 minutos). Não houve diferença $(\mathrm{P}>0,05)$ quanto à liberação de OT durante a ordenha.

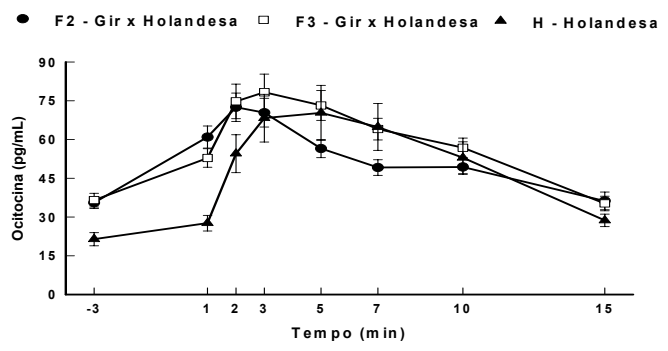

Figura 1. Concentração de ocitocina segundo os grupos raciais, durante a ordenha mecanizada exclusiva.

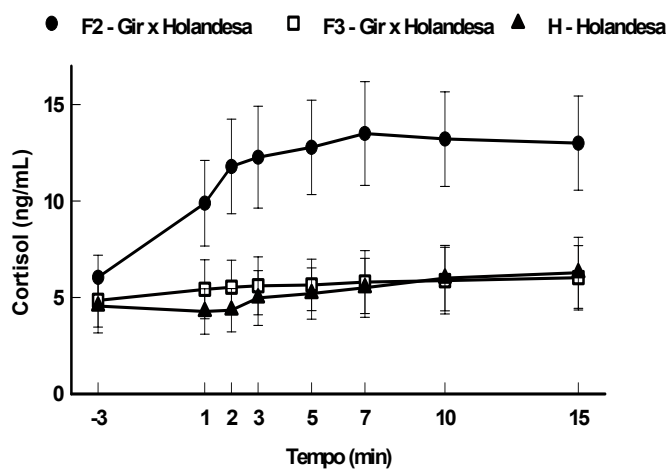

Figura 2. Concentrações de cortisol segundo os grupos raciais, durante ordenha mecanizada exclusiva.
O tempo de ordenha foi menor no grupo $\mathrm{H}$ (8,0 $\pm 0,5$ minutos) quando comparado aos dos grupos F3 $(9,4 \pm 0,2$ minutos $)$ e F2 $(10,0 \pm 0,3$ minutos). Embora as concentrações de base e da última amostra tenham sido influenciadas pelos grupos $(\mathrm{P}<0,05)$, a concentração total de OT, medida pela área sob a curva durante a ordenha, foi semelhante entre grupos $(\mathrm{P}>0,05)$.

Na Fig. 2, a concentração base de CORT foi semelhante para todos os grupos estudados, porém as concentrações de cortisol, medidas durante e após a ordenha, foram influenciadas pelos grupos $(\mathrm{P}<0,05)$. Os grupos $\mathrm{H}$ e $\mathrm{F} 3$ apresentaram perfis semelhantes de CORT, com pequeno aumento das concentrações durante $\mathrm{e}$ após a ordenha. O grupo F2 apresentou, desde o início da ordenha, altas concentrações de CORT (Fig. 2). Conseqüentemente, a concentração total de CORT, medida pela área sob a curva durante a ordenha, foi maior no grupo F2 $(\mathrm{P}<0,05)$.

A produção média de leite foi maior para o grupo $\mathrm{H}(25,6 \pm 4,3 \mathrm{~L} / \mathrm{vaca} / \mathrm{dia})$ seguido pelo grupo $\mathrm{F} 3$ $(16,2 \pm 1,7 \mathrm{~L} / \mathrm{vaca} / \mathrm{dia})$ e F2 $(13,1 \pm 1,0 \mathrm{~L} / \mathrm{vaca} / \mathrm{dia})$ $(\mathrm{P}<0,05)$. O leite residual foi influenciado pelos grupos, sendo F2 o grupo que apresentou a maior porcentagem de leite residual $(13,8 \pm 0,7 \%)$ comparado aos grupos $\mathrm{F} 3$ e $\mathrm{H}(9,5 \pm 1,0$ e $8,0 \pm 1,1 \%$, respectivamente). Portanto, a liberação de CORT foi inversamente correlacionada com a produção de leite. Observou-se correlação negativa $(\mathrm{P}<0,05)$ entre o tempo de liberação de OT e a porcentagem de leite residual (Tab. 1).

Tabela 1. Coeficientes de correlação entre produções de leite e de leite residual e liberações de ocitocina e cortisol durante a ordenha e liberação total

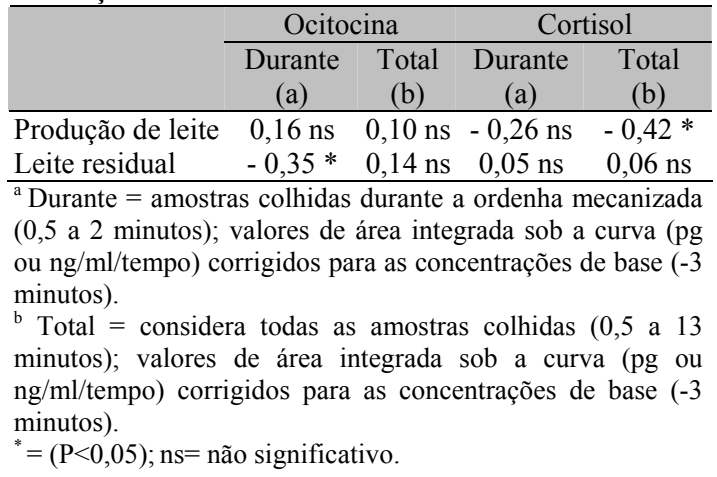


A classificação de comportamento dos três grupos raciais foi expressa em porcentagem. $\mathrm{O}$ grupo $\mathrm{H}$ manteve-se mais calmo durante a ordenha. Observou-se maior porcentagem de animais agitados no grupo F2 (Fig. 3). Os animais do grupo $\mathrm{H}$ foram manejados com maior facilidade quando comparados aos dos grupos F2 e F3, sendo o grupo F2 o mais difícil de se manejar durante a ordenha (Fig. 4). As ordenhas do grupo $\mathrm{H}$ foram realizadas com maior facilidade quando comparadas às dos grupos F2 e F3, sendo o grupo F2 mais difícil de ser ordenhado (Fig. 5).

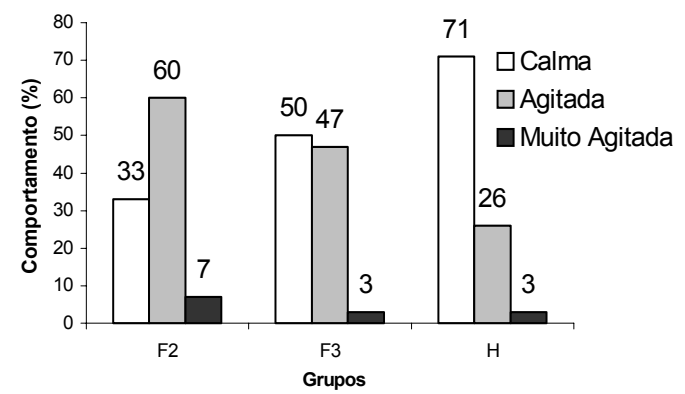

Figura 3. Comportamento segundo os grupos raciais, durante o manejo em sala de ordenha.

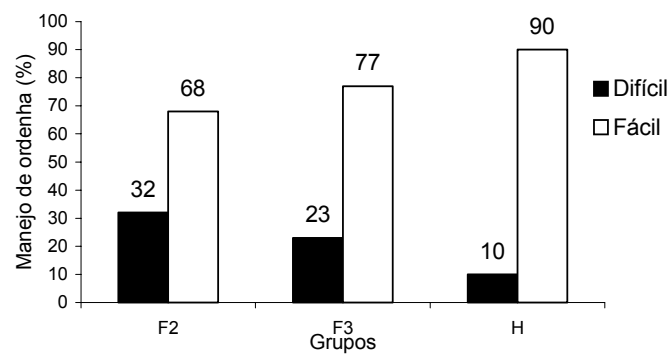

Figura 4. Dificuldade de manejo dos animais durante a ordenha segundo os grupos raciais.

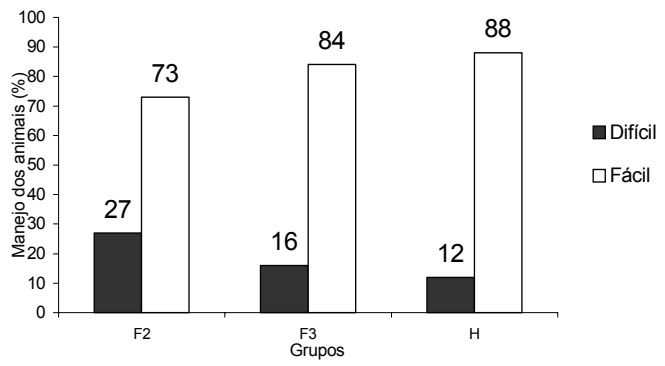

Figura 5. Dificuldade de realizar a ordenha mecânica exclusiva segundo os grupos raciais.

\section{DISCUSSÃO}

No grupo $\mathrm{H}$, a concentração de base de OT foi semelhante aos valores descritos em estudos prévios (Gorewit et al., 1992; Bar-Peled et al., 1995). Nos grupos F2 e F3, as concentrações foram mais altas do que os resultados observados na literatura (Gorewit et al., 1992; Bar-Peled et al., 1995). Estes resultados sugerem que a liberação de OT pode ser influenciada não só por fatores de ambiente, mas também pelo grupo genético. Os resultados encontrados na literatura não compararam animais de raças diferentes, o que poderia justificar as diferenças observadas nos resultados deste experimento para os animais mestiços.

Em situações de estresse, a liberação de OT pode ser inibida durante a ordenha (Bruckmaier e Blum, 1996); nas condições deste experimento, houve um importante e significativo aumento nas concentrações de OT durante a ordenha em todos os grupos, semelhante aos resultados obtidos por outros autores que trabalharam com vacas consideradas bem adaptadas à ordenha (Gorewit et al., 1992; Bar-Peled et al., 1995), sugerindo que a mudança de manejo de ordenha não causou estresse. Simultaneamente, a quantidade total de OT, liberada em todos os grupos, foi semelhante aos valores descritos por outros autores (Gorewit et al., 1992; Bar-Peled et al., 1995). Porém, não é possível afirmar, apenas com base na liberação de OT, que um grupo adaptou-se melhor à ordenha que outro.

Em relação à liberação de CORT, não houve diferença entre as concentrações de base nos grupos. Estes resultados foram semelhantes aos observados por Gorewit et al. (1992) e Bruckmaier et al. (1993) que pressupõem que os animais não estavam sob efeito de agentes estressantes antes da ordenha. No grupo F2, as concentrações de CORT aumentaram no início da ordenha e permaneceram elevadas durante e após a ordenha, conseqüentemente, a quantidade total de CORT liberado foi maior nesse grupo do que nos grupos H e F3. Estes resultados sugerem que as primeiras ordenhas após a mudança de manejo foram estressantes apenas para o grupo F2, o que o classificaria como menos adaptado à ordenha mecanizada na ausência do bezerro ao pé. 
Alguns autores observaram que o estresse causado pelas primeiras ordenhas realizadas depois da desmama foi sempre acompanhado de deficiência na liberação de OT e distúrbios na ejeção do leite (Tancin et al., 1995; Negrão e Marnet, 2002). Porém, no grupo F2, houve significativa liberação de OT durante a ordenha, e a resposta à OT não foi diferente dos outros grupos.

As produções médias de leite nos grupos foram semelhantes aos resultados obtidos por Gorewit et al. (1992). Embora as vacas do grupo F2 tenham retido um volume alto de leite residual, resultado este também observado por Murugaiyah et al. (2001), a porcentagem de leite residual foi baixa quando comparada aos resultados observados em vacas leiteiras submetidas a diferentes situações de estresse (Tancin et al., 1995).

Os resultados sugerem que todos os grupos experimentais foram capazes de adaptar-se à ordenha mecanizada, pois, em todos os grupos genéticos estudados, houve vacas com melhor ejeção de leite que outras. Entretanto, o grupo F2 provavelmente requer um tempo maior para adaptação ao manejo de ordenha na ausência do bezerro. Assim, parece ser possível melhorar o volume de leite obtido durante a ordenha mecanizada exclusiva através da seleção de animais mais adaptados à ordenha mecanizada.

\section{REFERÊNCIAS BIBLIOGRÁFICAS}

ABUBAKAR, B.Y.; BUVANENDRAN, V., Lactation curves of Friesian-Bunaji crosses in Nigeria. Livest. Prod. Sci., v.8, p.11-19, 1981.

BAR-PELED, U.; MALTZ, E.; BRUCKENTAL, I. et al. Relationship between frequent milking or suckling in early lactation and milk production of high producing dairy cows. J. Dairy Sci., v.78, p.27262736,1995

BRUCKMAIER, R.M.; BLUM, J.W. Normal and disturbed milk removal in dairy cows. In: SYMPOSIUM ON MILK SYNTHESIS; SECRETION AND REMOVAL IN RUMINANTS, 1996. Proceedings... Berna: School of Veterinary Medicine, 1996. p.37-42.

BRUCKMAIER, R.M.; SCHAMS, K.; BLUM, J.W.
Milk removal in familiar and unfamiliar surroundings: concentrations of oxytocin; prolactin; cortisol and B endorphin. J. Dairy Res., v.60, p.449-456, 1993.

GOREWIT, R.C.; SVENNERSTEN, K.; BUTLER, W.R. et al. Endocrine responses in cows milked by hand and machine. J. Dairy Sci., v.75, p.443-448, 1992.

MADALENA, F.E.; TRIVEDI, K.R. Experience with performance recording of dairy cattle in Brazil. ICAR Tech. Ser., v.1, p.235-246, 1998.

MAGNABOSCO, C.U.; LOBO, R.B.; BEZERRA, L.A. et al. Estimate of genetic change in milk yield in a Gyr herd in Brazil. Rev. Bras. Genet., v.16, p.957965, 1993.

MARNET, P.G.; NEGRÃO, J.A. The effect of a mixed-management system on the release of oxytocin, prolactin and cortisol in ewes during suckling and machine milking. Reprod. Nutr. Dev., v.40, p.271-281, 2000 .

MARNET, P.G.; NEGRÃO, J.A.; LABUSSIERE, J. Oxytocin release and milk ejection parameters in dairy ewes during machine milking in natural and induced lactation. Small Rum. Res., v.28, p.183-191, 1998.

MURUGAIYAH, M.; RAMAKRISHNAN, P.; SHEIKL OMAR, A.R. et al. Lactation failure in crossbred Sahiwal Friesian cattle. J. Dairy Res., v.68, p.165-174, 2001.

NEGRÃO, J.A.; MARNET, P.G. Effect of calf suckling on oxytocin, prolactin, growth hormone and milk yield in crossbred Gir x Holstein cows during milking. Reprod. Nutr. Dev., v.42, p.373-380, 2002.

NUTRIENT requirements of dairy cattle. 7.ed. Washington: NRC, 2001. 381p.

SANDOVAL - CASTRO, C.A.; ANDERSON, S.; LEAVER, J.D. Influence of milking and restricted suckling regimes on milk production and calf growth in temperate and tropical environments. Anim. Sci., v.69, p.287-296, 1999.

USER'S guide: statistics. Cary, NC: SAS Institute, 2000.

SOUZA, E.M.; MILAGRES, J.C.; REGAZZI, A.J. et al. Genetic and environmental effects on milk yield in Gir dairy herds. Rev. Soc. Bras. Zootec., v.25, p.889901, 1996.

TANCIN, V.; HARCEK, L.; BROUCEK, J. et al. Effect of suckling during early lactation and changeover to machine milking on plasma oxytocin and cortisol levels and milking characteristics in Holstein cows. J. Dairy Res., v.62, p.249-256, 1995. 